\title{
Betriebseinrichtungen mit Sicherheitsfunktion im PLS
}

Namur AK 4.5 Funktionale Sicherheit - Subteam NE165

In der zweiten Ausgabe der IEC 61511 sowie der überarbeiteten VDI/VDE 2180 werden Maßnahmen zur Umsetzung von risikoreduzierenden Funktionen im Leitsystem beschrieben. Im vorliegenden Artikel wird eine mögliche Implementierung dieser Maßnahmen im betrieblichen Leitsystem erörtert.

SCHLAGWÖRTER Sicherheitsfunktion / PLT-BS

Safety functions in the BPCS -

Implementing rules from the IEC 61511 and VDI/VDE 2180 standards

The second edition of IEC 61511 and the revised VDI/VDE 2180 describe measures for implementing risk reduction functions in a basic process control system (BPCS). This paper discusses a possible implementation of these safety measures together with the operational BPCS.

KEYWORDS safety function / BPCS protection layer 
THOMAS GABRIEL, Covestro

DIRK HABLAWETZ, BASF

MICHAEL KARTENBERG, Evonik Technology \& Infrastructure

MICHAEL KRAUSS, BASF

UDO MENCK, Dow

GREGOR SCHMITT-PAUKSZTAT, Bayer

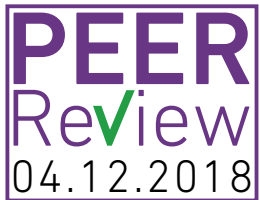

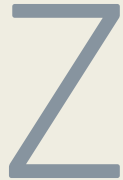

ur Reduzierung von prozesstechnischen Risiken gibt es in der Prozessindustrie verschiedene Möglichkeiten. Unabhängig vom Bewertungsverfahren (zum Beispiel Risikograph, Risikomatrix, LOPA) werden im Rahmen von Sicherheitsbetrachtungen üblicherweise Maßnahmen zur Risikoreduzierung definiert. Die notwendige Risikoreduzierung bis zu einem tolerierbaren Risiko (oft beschrieben in sogenannten Risikoreduktionsfaktoren, RRF) kann durch einzelne Maßnahmen, aber auch durch eine Kombination verschiedener Maßnahmen beziehungsweise Schutzebenen mit unterschiedlicher Risikoreduktion erfolgen. IEC 615115-3 (siehe [1]) beschreibt dieses Konzept ausführlich.

In Deutschland wird oft nur eine einzige Maßnahme/ Schutzebene, zum Beispiel ein Sicherheitsventil oder eine PLT-Sicherheitseinrichtung, zur Risikoreduzierung verwendet. Manchmal sind auch Risikobewertungsverfahren so ausgelegt, dass sie ausschließlich zu einer einzelnen PLT-Sicherheitsfunktion führen und damit andere sinnvolle Maßnahmen-Kombinationen von vornherein ausschließen.

In der IEC 61511-1 (siehe [2]) werden neben PLTSicherheitseinrichtungen auch andere sicherheitstechnische Maßnahmen auf der Basis von Prozessleittechnik beschrieben. Diese über die betrieblichen Notwendigkeiten hinausgehenden PLT-Betriebseinrichtungen werden dort als Schutzebene - im Englischen als BPCS Protection Layer - bezeichnet. Außerdem werden in IEC 61511-1 Anforderungen an diese Maßnahmen gestellt. Im vorliegenden Beitrag werden genau diese risikomindernden Maßnahmen beschrieben, die in einem Prozessleitsystem (PLS) implementiert werden können. Diese Maßnahmenklasse kann maximal eine Risikoreduzierung von 10 (RRF 10) zur gesamten Risikoreduzierung beitragen oder diese bei einer nur niedrigen notwendigen Risikoreduzierung gegebenenfalls auch vollständig abdecken. In der aktuell überarbeiteten VDI/ VDE 2180 (siehe [3]) wird diese Maßnahmenklasse ebenfalls aufgegriffen und als PLT-Betriebseinrichtung mit Sicherheitsfunktion (PLT-BS) bezeichnet - daher wird im Text ausschließlich von PLT-BS gesprochen. Nachfolgend wird beschrieben, wie PLT-BS in einem PLS umgesetzt werden können. Herausfordernd ist dabei die Umsetzung in einem PLS, das gleichzeitig zur betrieblichen Steuerung von Prozessen verwendet wird.

\section{NORMATIVE ANFORDERUNGEN AN PLT-BS}

In der IEC 61511-1 werden Anforderungen an PLTBS gestellt. Dabei sind in der zweiten Ausgabe der IEC 61511-1 zusätzliche Hinweise an die Unabhängigkeit dieser Maßnahmen gegeben. Prinzipiell können PLT-Betriebseinrichtungen mit Sicherheitsfunktion zu einer Risikoreduzierung bis Faktor 10 eingesetzt werden (siehe Tabelle 1).

Während die Realisierung von Maßnahmen mit SIL 1 bis SIL 3 in IEC 61511 umfassend beschrieben ist, werden zu PLT-BS nur wenige konkrete Hinweise gegeben.

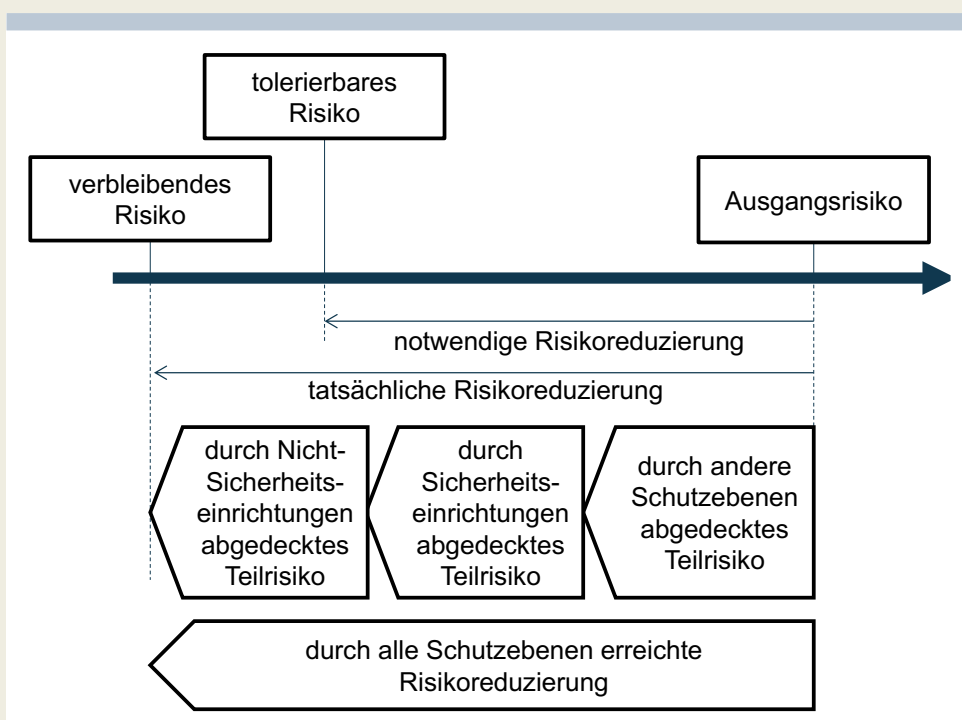

BILD 1: Mögliche Maßnahmen/Schutzebenen zur Risikoreduzierung 
TABELLE 1: Risikoreduzierungsfaktoren in Anlehnung an IEC 61511-3

\begin{tabular}{|l|l|}
\hline PLT-Sicherheitsfunktion & Risikoreduzierungsfaktor RRF \\
\hline SIL 3 & $>1000$ \\
\hline SIL 2 & $>100$ \\
\hline SIL 1 & $>10$ \\
\hline PLT-BS & $\leq 10$ \\
\hline
\end{tabular}

Neu in Teil 1 der zweiten Ausgabe von IEC 61511-1 sind explizite Anforderungen an die Kombination von PLT-BS bei gleichem abzudeckenden Risiko und Anforderungen an die Unabhängigkeit der Maßnahme.

So darf nach IEC 61511-1 nicht mehr als eine PLT-BS für die gleiche Abfolge von Ereignissen, die zum gefährlichen Ereignis führen, verwendet werden, falls die PLT-Betriebseinrichtung Auslöser für eine Anforderung an die Schutzebene ist, oder es dürfen nicht mehr als zwei PLT-BS für die gleiche Abfolge von Ereignissen, die zum gefährlichen Ereignis führen, verwendet werden, falls die PLT-Betriebseinrichtungen nicht Auslöser für eine Anforderung ist.

Außerdem muss jede PLT-BS unabhängig und getrennt von der auslösenden Quelle und jeder anderen PLTBS sein, sodass die geforderte Risikominderung jeder PLT-BS nicht beeinträchtigt wird. Dabei wird explizit darauf verwiesen, dass eine PLT-Betriebseinrichtung die auslösende Quelle sein kann.

Prinzipiell führen diese Anforderungen dazu, dass PLT-BS unabhängig von betrieblichen Einrichtungen (PLT-B) realisiert werden müssen. Analog zur Darstellung von unabhängigen Schutzebenen aus IEC 61511-2 wären damit PLT-Einrichtungen vollständig getrennt aufzubauen (siehe Bild 2).

Weiterhin werden in IEC 61511-2 Anforderungen an den Umgang mit PLT-BS gestellt, die in VDI/VDE 2180 wieder aufgegriffen werden. Daraus ergibt sich, dass PLT-BS grundsätzlich wie jede Sicherheitsfunktion in einem Sicherheitslebenszyklus zu handhaben sind und damit ähnlichen Anforderungen bezüglich des Managements der funktionalen Sicherheit solcher Maßnahmen unterliegen.

\section{Management der funktionalen Sicherheit}

Damit ist prinzipiell das Modell des Lebenszyklus anwendbar. Die Anforderungen an alle Lebenszyklusphasen sind IEC 61511 beziehungsweise VDI/VDE 2180 zu entnehmen. Die Basis für das Management der funktionalen Sicherheit bilden dabei:

- Regelung von Verantwortlichkeiten und

Qualifikationen,

- Änderungsmanagement,

- Management von Verifikationstätigkeiten.

\section{Spezifikation der Sicherheitsanforderungen}

Die funktionalen Anforderungen an PLT-BS müssen spezifiziert werden. Hierzu gehören beispielsweise

- Schalt-/Alarmpunkte,

- beteiligte Sensoren/Aktoren,

- Architektur/Redundanz,

- Messbereich und -genauigkeit,

- Prozesssicherheitszeit,

- Prozessanschluss- und Medieneigenschaften,

- Anforderungen an Überbrückungen,

- Verhalten bei Wiederanlauf nach Energieausfall, beziehungsweise USV-Konzept,

- Konzept für wiederkehrende Prüfung/Prüfkonzept. Leitsysteme haben in der Regel kein vordefiniertes Ausfall-/Wiederanlaufverhalten. Für die Spezifikation von PLT-BS im Leitsystem ist dies zu berücksichtigen.

\section{Generelle Anforderungen}

Für die Implementierung von PLT-BS ist auf etablierte Ingenieurspraxis zu achten. PLT-BS müssen immer Vorrang vor anderen betrieblichen Funktionen haben. So darf zum Beispiel ein Ventil, das über eine PLT-BS geschlossen ist, nicht durch eine betriebliche Funktion geöffnet werden. Änderungen im Leitsystem an PLT-B dürfen keinen Einfluss auf PLT-BS haben können (zum Beispiel durch Verwechslung der zu ändernden Funktion). PLT-BS sind geeignet zu kennzeichnen.

Strukturell sind einkanalige Systeme für PLT-BS ausreichend - die Hardwarefehlertoleranz ist also mindestens Null. Für PLT-BS ist kein rechnerischer Verfügbarkeitsnachweis notwendig, wie bei SIL 1 bis SIL 4 .

\section{Anforderungen an Sensor- und Aktor-Teilsystem}

Die in PLT-BS eingesetzten Sensoren und Aktoren müssen mindestens einschlägigen Industriestandards entsprechen. Um eine Verfügbarkeit der Sicherheitsfunktion so zu gewährleisten, dass eine Risikoreduzierung von zehn möglich wird, werden betriebsbewährte Geräte im Sinne IEC 61511-1 und/oder nach IEC 61508 entwickelte Geräte empfohlen.

\section{Montage, Inbetriebnahme und Validierung}

Den Phasen des Sicherheitslebenszyklus folgend, müssen mit Blick auf Montage, Inbetriebnahme und Validierung die in einer PLT-BS verwendeten Feldgeräte gemäß Spezifikation installiert und in Betrieb gesetzt werden. Die fachgerechte Installation und Inbetriebnahme ist geeignet zu dokumentieren und zu verifizieren. Dazu kann zum Beispiel das Inbetriebnahmeprotokoll im Sinne des Vieraugenprinzips von einer zweiten Person ausgefüllt werden.

Analog gilt für die Steuerung als Logik-Teilsystem einer PLT-BS, dass diese gemäß Spezifikation installiert und in Betrieb gesetzt wird. Die korrekte Umsetzung 
PLT-B

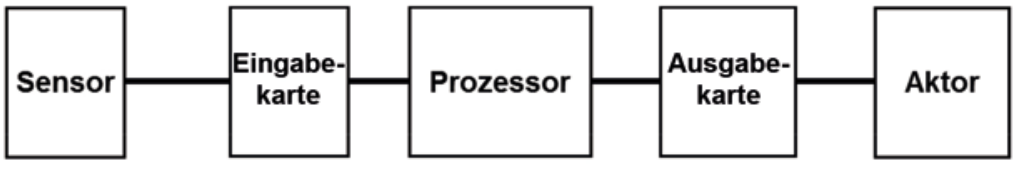

PLT-BS

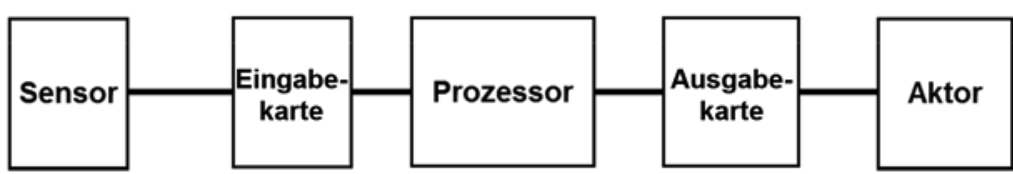

BILD 2: Vollständig getrennte PLT-Betriebseinrichtung und PLT-Betriebseinrichtung mit Sicherheitsfunktion

der Steuerungslogik ist geeignet zu verifizieren. Dies kann zum Beispiel durch eine Steuerungssimulation im Rahmen eines FAT erfolgen.

Die spezifikationsgemäße Funktionsweise der PLT-BS ist in geeigneter Form im Rahmen einer Validierung nachzuweisen. Entscheidend für die Validierung ist, dass eine geeignete Funktionsprüfung unter Einbeziehung der gesamten Wirkkette (Sensor, Logik, Aktor) durchgeführt wird. Gegebenenfalls sind verschiedene Betriebszustände zu berücksichtigen.

\section{Betrieb der PLT-BS}

Komponenten, die in PLT-BS verwendet werden, müssen vor Inbetriebnahme und in geeigneten Abständen wiederholend sowie nach Modifikation auf Basis der spezifizierten Sicherheitsfunktion geprüft und die Ergebnisse dokumentiert werden. Hierzu gehören Sensor-, Logik- und Aktor-Teilsystem. Ein Konzept zur regelmäßigen Prüfung ist durch den Betreiber festzulegen. Abhängig von zu erwartenden Fehlern oder den Erfahrungen im späteren Betrieb kann das Prüfintervall verkürzt oder verlängert werden.

Prüfungsumfang, -durchführung und -dokumentation sowie das Intervall der Wiederholungsprüfung können sich am Umgang mit PLT-Sicherheitseinrichtungen (SIL 1 bis SIL 4) orientieren und beinhalten die gesamte Sicherheitsfunktion von Sensor- über Logik- bis Aktor-Teilsystem. Analog zu PLT-Sicherheitseinrichtungen sind auch zeitlich versetzte Prüfungen von Teilsystemen zulässig.

Zur Sicherstellung, dass die gewünschte Risikoreduzierung der PLT-BS im Betrieb erreicht wird, sollte ein Nachweis geführt werden. Dazu hat es sich bewährt, Ausfälle von PLT-BS zu registrieren und zu analysieren. Als Hilfsmittel können statistische Auswertungen wie zum Beispiel über Namur.smart genutzt werden (siehe auch NE 93).

Der Umgang mit Störungen an PLT-BS ist organisatorisch festzulegen. Hierzu gehören zum Beispiel die maximale Zeit, die eine PLT-BS außer Betrieb sein darf sowie vordefinierte Ersatzmaßnahmen.
Wird ein gefährlicher Ausfall einer PLT-BS erkannt, muss eine Ursachenuntersuchung stattfinden, um den Grund des Ausfalls zu ermitteln. Darauf sind dann Gegenmaßnahmen abzuleiten, die einen derartigen Ausfall zukünftig möglichst unterbinden.

Wenn PLT-BS zeitlich begrenzt überbrückt werden sollen, muss dafür ein Konzept vorliegen. Dieses Konzept kann zum Beispiel im Rahmen einer Sicherheitsbetrachtung erstellt werden. In diesem Rahmen ist auch festzulegen, welche Freigaben für eine Überbrückung von PLT-BS erforderlich sind. Während der Zeit einer Überbrückung müssen Ersatzmaßnahmen definiert werden, die mindestens dieselbe Risikoreduzierung erzielen, wie die überbrückte PLT-BS.

\section{Änderungsmanagement}

PLT-BS unterliegen einem Änderungsmanagement. Ein solches Änderungsmanagement muss mindestens eine festgelegte Vorgehensweise hinsichtlich der Freigabe zur Erstellung beziehungsweise Änderung enthalten sowie die Anfertigung beziehungsweise Fortschreibung der Spezifikation der Sicherheitsfunktion beschreiben.

\section{REALISIERUNG DES LOGIK-TEILS VON PLT-BS}

Anders als zum Beispiel im amerikanischen Umfeld ist es in Deutschland unüblich, mehr als ein PLS zu implementieren, um damit zwei unabhängige Schutzebenen wie nach Bild 2 zu bilden. Typischerweise sind ein betriebliches PLS und gegebenenfalls ein separates Sicherheitssystem (SIS) in Anlagen implementiert.

Damit stellt sich die Frage, wie die Anforderung der Unabhängigkeit zwischen PLT-BS und PLT-B aus IEC 61511-1 umgesetzt werden kann.

Prinzipiell könnten die PLT-BS im Sicherheitssystem realisiert werden. Damit sind sie unabhängig von PLT-B und die normative Grundanforderung wäre erfüllt.

Weiterhin wäre die Umsetzung im sicherheitsgerichteten Teil von kombinierten PLS/SIS denkbar. Es gelten 


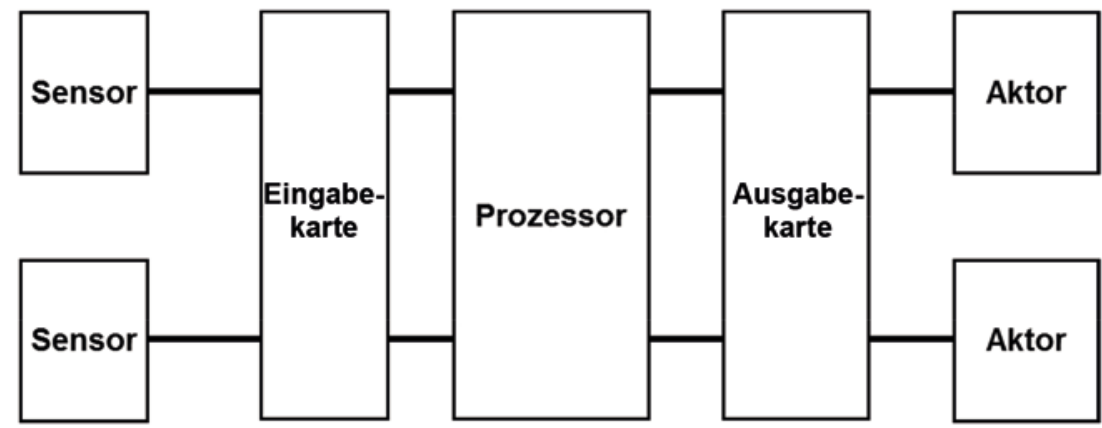

BILD 3: Gemeinsam genutzte Komponenten von PLT-Betriebseinrichtung und PLT-Betriebseinrichtung mit Sicherheitsfunktion

ähnliche Bedingungen wie bei der Implementierung in einem separaten SIS.

Es ist aber auch die Realisierung von PLT-BS im betrieblichen PLS denkbar, gemeinsam mit anderen PLT-B. Normativ gestützt wird diese Vorgehensweise durch die Öffnungsklausel, dass PLT-BS und PLT-B nicht zwingend vollständig getrennt sein müssen, sondern insoweit unabhängig, dass die geforderte Risikominderung jeder PLT-BS nicht beeinträchtigt wird. Laut IEC 61511-1 ist die Unabhängigkeit zu bewerten.

\section{Logik-Teil der PLT-BS im PLS}

Bei Nutzung eines gemeinsamen Logik-Teilsystems für PLT-BS und PLT-B müssen die Anforderungen an die hinreichende Unabhängigkeit im Rahmen der erforderlichen Risikoreduzierung genauer betrachtet werden. Falls Komponenten von beiden Ebenen gleichzeitig genutzt werden, ergeben sich zusätzliche Fehler mit gemeinsamer Ausfallart (commonmode-Fehler). Damit ist die Risikoreduzierung der einzelnen Schutzebene prinzipiell erst einmal beeinträchtigt. Es ergibt sich - je nach Verschaltung von Komponenten - prinzipiell eine Struktur wie in Bild 3 dargestellt.

Die Beeinträchtigung der Risikoreduzierung der Schutzebene PLT-BS wird nur vernachlässigbar, wenn alle relevanten Common-mode-Fehler der gemeinsam genutzten Komponenten gefunden werden und kompensierende Maßnahmen vorhanden sind. Die gegebenenfalls erforderlichen zusätzlichen kompensierenden Maßnahmen müssen entweder unterbinden, dass die Common-mode-Fehler auftreten oder im Fall eines auftretenden Fehlers dafür sorgen, dass der sichere Zustand des Systems erreicht werden kann.

Damit ist eine ausreichende Risikoreduzierung bezogen auf vollständig getrennte Ebenen erreicht, wenn die gemeinsam genutzten Komponenten den im Folgenden beschriebenen Eigenschaften genügen.

\section{Anforderungen an gemeinsame Logik-Komponenten in PLT-B und PLT-BS}

Prinzipiell werden in IEC 61511 keine Anforderungen an die Hardwarekomponenten eines Leitsystems gestellt, das als Logik-Teilsystem einer PLT-BS genutzt wird. Durch die im Folgenden beschriebenen zusätzlichen Anforderungen an das Leitsystem und daraus gegebenenfalls resultierenden Maßnahmen kann gerechtfertigt werden, dass die gewünschte Risikoreduzierung nicht beeinträchtigt wird. Die Hersteller der für PLT-BS genutzten Systeme müssen über ein Qualitätssicherungssystem zum Beispiel gemäß DIN ISO $9000 \mathrm{ff}$. verfügen und die Anforderungen für eine Verwendung in kontinuierlich betriebenen Prozessanlagen erfüllen. In der Regel kommen in der Prozessindustrie nur Systeme global etablierter Hersteller zum Einsatz.

Jede gemeinsam von PLT-BS und PLT-B genutzte Logik-Komponente muss mindestens eine der folgenden Anforderungen erfüllen:

- Es ist eine Redundanz dieser Komponente vorhanden, oder

- die mittlere Betriebszeit bis zum Auftreten eines Fehlers (MTTF) jeder für die PLT-BS erforderlichen Komponente beträgt 100 Jahre. Die Zuverlässigkeit kann entweder über den Hersteller bestätigt werden (zum Beispiel FMEDA) oder durch Störstatistiken belegt werden (zum Beispiel Namur.smart), oder

- jeder Fehler dieser Komponente (Spannungsversorgung, Kommunikationsbaugruppen, Prozessor, Eingabe-/Ausgabekarte et cetera) führt zu einem sicheren Zustand der Anlage.

\section{Fehlermodi von gemeinsamen Logik-Komponenten} für PLT-B und PLT-BS

Je nach Art der gemeinsam genutzten Komponente als PLT-BS und PLT-B (siehe Bild 3) sind spezifische Fehlermodi möglich, deren Kompensation mit unterschiedlichen Maßnahmen erreicht werden kann. 
Zur Identifizierung möglicher Common-mode-Fehler wurde gemeinsam mit Herstellern von Leitsystemen beziehungsweise Sicherheitssystemen eine Fehleranalyse durchgeführt und gegebenenfalls erforderliche Maßnahmen identifiziert, die zu der Annahme führen, dass eine Risikoreduzierung von zehn für PLT-BS erreicht werden kann.

Die Ergebnisse werden in der Namur-Empfehlung NE 165 (siehe [4]) veröffentlicht. Dort werden mögliche Fehler mit darauf abgestimmten Maßnahmenpaketen beschrieben, sodass der Anwender je nach Struktur des Leitsystems die geeigneten Pakete anwenden kann.

Da die Identifizierung von Common-mode-Fehlern abhängig ist von der Struktur des PLS, können Fehlermodi nur prinzipiell erläutert werden. Nachfolgend ist eine möglichst generische aber willkürliche Untergliederung von Fehlermodi vorgenommen worden, die naturgemäß das Potenzial doppelter Adressierung von Fehlern hat. Gegenmaßnahmen zur Kompensation von Fehlern sind durch den Anwender zu implementieren oder durch den Hersteller auszuschließen. Vorschläge zu möglichen Gegenmaßnahmen können NE 165 entnommen werden.

\section{Allgemeine Fehlermodi}

Prozessleitsysteme sind in der Regel auf Anlagenverfügbarkeit ausgelegt. Daher ist neben der Spezifikation der Komponente der PLT-BS für das Umfeld der Prozessindustrie mit Berücksichtigung der Umgebungseinflüsse vor allem wichtig, dass ein undefiniertes Verhalten zum Beispiel durch Energieverlust bei der PLT-BS verhindert wird. Nach einem Firmware- oder SystemsoftwareUpdate ist sicherzustellen, dass nicht eine oder mehrere PLT-BS beeinflusst sind oder fehlerhaft arbeiten.

\section{Fehlermodi Logiksystem (Hardware)}

Alle gemeinsam von PLT-BS und PLT-B genutzten Hardwarekomponenten (zum Beispiel Prozessoren, Switch, Server) können fehlerbehaftet sein. Typische Fehler können hardwarebedingte Fehler sein wie Bit-Flip oder zufälliger Hardware-Fehler oder Speicherfehler. Weiterhin könnte eine nicht spezifikationsgerechte Versorgungsspannung zu undefiniertem Verhalten führen (zum Beispiel durch Spannungsspitzen). Außerdem sind softwarebedingte Fehler möglich, wie zum Beispiel Programmfehler, die einen undefinierten Zustand auslösen (zum Beispiel Division durch Null). Zu den softwarebedingten Fehlern gehören auch potenzielle Fehler beim Download von Programmen vom Engineering-System auf die Logikverarbeitung oder aber ein unbeabsichtigter Stopp der CPU.

\section{Fehlermodi Kommunikationsweg}

Der Signalpfad einer PLT-BS in einem PLS ist potenziell fehlerbehaftet. Die Betrachtung bezieht sich auf sämtliche Kommunikationswege des Logik-Teilsystems (zum Beispiel von Remote-I/O über CPU zur nächsten Remote-I/O). Mögliche Fehler sind hier unter anderem, dass das Kommunikationsmedium defekt ist. Dazu zählen unter anderem Kurzschluss, Kabelbruch, Steuerluftausfall et cetera. Der Kommunikationspartner könnte aber auch falsch konfiguriert sein, nicht antworten oder ungefragt Signale absetzen. Die Kommunikation könnte überlastet sein, zum Beispiel bei Bus-Kommunikation. Besondere Aufmerksamkeit gilt insbesondere der Zusammenschaltung von Komponenten verschiedener Hersteller bezüglich Inkompatibilitäten in der Kommunikation mit Fremdsystemen. Prinzipiell sind auf dem Kommunikationsweg Datenverfälschungen möglich, sodass Informationen zum Beispiel in der falschen Reihenfolge ankommen oder Bit-Flips auftreten.

\section{Fehlermodi Ein-Ausgabe-Komponenten}

Neben den schon aufgeführten Fehlermodi, wie Fehler in der Programmabarbeitung zum Beispiel durch Bit-Flip, Bit-Stuck oder interner Hardware-Fehler der Baugruppe, ist bei Ein-Ausgabekomponenten insbesondere bei abgesetzten Kommunikationsbaugruppen zu bewerten, dass ein unbemerkter Energieverlust oder Verbindungsabbruch auftreten können. Hier sind entsprechende Diagnosemaßnahmen sinnvoll. Bei AnalogEingangs-Karten mit nur einem Analog-Digital-Wandler mit Multiplexer ergibt sich als potenzieller Fehler, dass unbemerkt der Analog-Eingang zu viel oder zu wenig anzeigt (zum Beispiel durch eine Drift).

\section{Fehlermodi Human Machine Interface (HMI)}

Sofern die Aktion einer PLT-BS durch einen Bediener ausgeführt werden soll, ist die Alarmierung mit Anzeige sowie gegebenenfalls die Schnittstelle zur vorhandenen Aktorik ebenfalls im sicherheitskritischen Pfad der PLT-BS. Falls die Alarmierung über eine optische Anzeige (Leuchtmelder) erfolgt, kann diese analog zu allen anderen Aktoren betrachtet werden. Falls die Alarmierung mit einem HMI erfolgt, sind mögliche Fehler zu bewerten. Dazu gehört, dass die Visualisierung für PLT-BS-Alarme nicht mehr aktualisiert wird oder PLT-BS-Alarme nicht im HMI ankommen. Bei der Darstellung sollte darauf geachtet werden, dass der Alarm auch als Teil einer PLT-BS erkannt werden kann.

\section{Fehlermodi Peripherie}

Die an das PLS (zum Beispiel an den Anlagenbus) angeschlossene Peripherie (zum Beispiel Datensammler, Plant-Informations- und Management-Systeme) kann potenziell die PLT-BS beeinflussen. Diese mit dem PLS verbundenen Systeme sind bezüglich potenzieller Fehler auf die PLT-BS zu bewerten. Hauptursachen für 


\section{REFERENZEN}

[1] IEC 61511-3:2016. (2016). Functional safety - Safety instrumented systems for the process industry sector Part 3: Guidance for the determination of the required safety integrity levels. IEC: www.iec.ch

[2] IEC 61511-1:2016+AMD1:2017 CSV Consolidated version (2017). Functional safety - Safety instrumented systems for the process industry sector - Part 1: Framework, definitions, system, hardware and application programming requirements. IEC: www.iec.ch

[3] VDI/VDE 2180 Blatt 1:2018-02. (2018). Funktionale Sicherheit in der Prozessindustrie, Entwurf. VDI: www.beuth.de

[4] NAMUR. (o.J.). NE 165: PLT-Betriebseinrichtungen mit Sicherheitsfunktion, in Vorbereitung. NAMUR: www. namur.net

[5] NAMUR. (2017). NA 163: IT-Risikobeurteilung von PLT-Sicherheitseinrichtungen. NAMUR: www.namur.net

Fehler sind, dass Systeme aus der Peripherie auf PLT-BS Informationen schreiben und die Sicherheitsfunktion beeinträchtigen. Außerdem könnten Systeme der Peripherie den Kommunikationsweg überlasten.

\section{Fehlermodi Engineering}

PLT-BS unterliegen einem Änderungsmanagement. Insbesondere bei kombinierten Architekturen von PLTBS und PLT-B in einem System muss ein wirksames Änderungsmanagement sichergestellt werden. Das Änderungsmanagement muss ebenfalls die Rückwirkungsfreiheit von Änderungen im Leitsystem auf die PLT-BS sicherstellen. Änderungen in PLT-B dürfen sich nicht auf PLT-BS auswirken.

In der Regel gehen die nachfolgend dargestellten Fehler über die Betrachtung eines Standard-Änderungsmanagements hinaus. Dennoch sollten sie in Betracht gezogen und Gegenmaßnahmen ergriffen werden.

Wesentliche Fehlerquellen im Engineering umfassen zum Beispiel die unautorisierte oder versehentliche Änderung von Software beziehungsweise die Konfiguration über Engineeringstation oder die Bedienebene. Beispielsweise könnte eine PLT-BS fälschlicherweise anstatt einer geplanten Änderung an einer betrieblichen Funktion modifiziert werden oder eine versehentliche Übertragung des Programms auf die Logikeinheit erfolgen (Beispiel: statt Teilanlage die gesamte Anlage neu geladen). Es könnte eine unbemerkte De-Synchronisation verschiedener Programmstände PLT-BS/PLT-B auftreten, zum Beispiel bei Vergabe von Änderungen in Teilanlagen an Externe und Rückspielen des Codes, wobei während der externen Änderungen das LiveSystem geändert wurde.

Insbesondere für den Fall, dass Hardwarekomponenten aber auch Software nicht als sicherheitsrelevant beziehungsweise Teil von PLT-BS zu erkennen sind, können Fehler in der Handhabung auftreten. Damit könnte fehlerhaft die PLT-BS keine Priorität vor betrieblichen Funktionen haben. Die PLT-BS könnte unbemerkt dauerhaft gebrückt sein. Notwendige Failsafe-Konfigurationen könnten nicht beachtet sein.

Beim Engineering der Software von PLT-BS können Fehler in der Abarbeitung aufgrund komplexer Codestrukturen entstehen, wie der ungeschützte beziehungsweise übergreifende Schreibzugriff betrieblicher Funktionen auf PLT-BS (zum Beispiel Mehrfach-Zugriff auf I/O-Ebene oder Variablen). Außerdem könnte der Abarbeitungs-Zyklus der Software unbemerkt nicht durchlaufen werden. Damit werden Programmteile nicht abgearbeitet und zum Beispiel Prozesswerte nicht eingelesen und aktualisiert. Weiterhin könnte die CPU überlastet werden oder auch die Kommunikation und unbemerkt die Reaktionszeit von Sensor-Teilsystem bis Aktor-Teilsystem der PLT-BS verlängert werden.

\section{Cyber Security}

Prinzipiell sind PLT-BS wie alle Sicherheitsfunktionen auch bezüglich der Manipulation von außen zu bewerten. Mögliche Fehlermodi aus dem Bereich Cyber Security sind separat zu betrachten (zum Beispiel analog NA 163, siehe [5]). Dabei ist zu berücksichtigen, dass PLT-BS im Verhältnis zu Sicherheitsfunktionen SIL 1 bis SIL 4 ein geringeres Risiko kompensieren. Dementsprechend können Maßnahmen bezüglich Cyber Security angepasst werden.

\section{Bewertung}

Mit Hilfe der aufgeführten Fehlermodi kann spezifisch für jedes PLS eine Bewertung vorgenommen werden, welche Fehler bei gemeinsamer Nutzung von Komponenten als PLT-BS und PLT-B entstehen. Aus den Fehlermodi können Gegenmaßnahmen abgeleitet werden. Jeder Anwender kann für sein Leitsystem prinzipiell so vorgehen, dass zunächst die Struktur des Leitsystems festgestellt wird. Danach werden von PLT-BS und PLT-B gemeinsam genutzte Komponenten identifiziert. Dann wird die Zuverlässigkeit der Hardware sichergestellt (Auswahl der Hardware). Anschließend können je nach gemeinsam genutzter Komponente Gegenmaßnahmen für potenzielle Fehler implementiert werden.

Auf diese Weise kann auch bei gemeinsamer Nutzung von Komponenten als PLT-BS und PLT-B eine ausreichende Risikoreduzierung der Schutzebene PLT-BS erreicht werden.

\section{FAZIT UND AUSBLICK}

Die beschriebene Vorgehensweise liefert eine Möglichkeit, Maßnahmen mit einer Risikoreduzierung bis zu zehn (PLT-BS) im betrieblichen Leitsystem zusammen mit 
anderen betrieblichen Funktionen (PLT-B) zu implementieren. Damit werden die Anforderungen aus IEC 61511 bezüglich der Bewertung der Unabhängigkeit von PLTBS und PLT-B erfüllt.

Eine detaillierte Ausarbeitung der Vorgehensweise sowie Beispiele für mögliche Gegenmaßnahmen zur Kompensation von potenziellen Fehlern gemeinsam genutzter Komponenten liefert die NE 165.

Die konkrete Implementierung der PLT-BS ist abhängig von der Struktur des jeweiligen Leitsystems des Anwenders. Die Wahl der Implementierung wird zusätzlich von verschiedenen Randbedingungen wie Investitionsund Instandhaltungskosten abhängen.

Für künftige Anwendungen wünschen sich die Betreiber von Prozessleitsystemen mehr Unterstützung von den Herstellern bei der Implementierung von PLT-BS. Viele der im Text diskutierten Fehlermodi können durch herstellerseitige Maßnahmen kompensiert werden. Im
Wesentlichen geht es um eine Bestätigung der Hardwarezuverlässigkeit und eine Kapselung der PLT-BS innerhalb der PLT-B-Logik, um eine hinreichende Unabhängigkeit von PLT-BS und PLT-B zu erreichen. Dies würde die Implementierung vereinfachen und den täglichen Umgang mit solchen Anordnungen sicherer gestalten. Um dieses Konzept erfolgreich zu implementieren, erwarten die Endanwender klare Implementierungshinweise, die in der neuen NE 165 konkretisiert werden. Lieferanten, die diese Anforderungen erfüllen, können Konformität mit der NE 165 deklarieren. Dieses kann den Endanwendern bei der Auswahl zukünftiger Systeme helfen.

Abschließend möchten wir allen Diskussionspartnern, Herstellern sowie Betreibern für die wertvollen Beiträge aus unseren Workshops - insbesondere den Ergebnissen aus den Workshops der Namur-Hauptsitzungen 2017 und 2018 - zu diesem Thema herzlich danken.

\section{AUTOREN}

Dr. THOMAS GABRIEL (geb. 1980) leitet die Fachgruppe ,Funktionale Sicherheit' in der globalen PLT-Organisation bei Covestro. In dieser Rolle ist er zuständig für die globale konzerninterne Regelwerksetzung und strategische Ausrichtung, sowie die Bereitstellung von Werkzeugen und Services rund um die Funktionale Sicherheit.

Dipl.-Ing. DIRK HABLAWETZ (geb. 1964) leitet in der BASF SE die Einheit Business and Safety Excellence im globalen Bereich Engineering und Technical Expertise und ist Leiter des globalen Expert Teams für Funktionale Sicherheit. Derzeit steht die Kombination von Anlagensicherheit und Cyber-Sicherheit und deren gegenseitige Abhängigkeit im Fokus seiner Tätigkeit. Dirk Hablawetz vertritt die BASF in nationalen und internationalen Gremien auf dem Gebiet der Funktionalen Sicherheit.

Dipl.-Ing. MICHAEL KARTENBERG (geb. 1964) leitet in der Evonik Technology \& Infrastructure $\mathrm{GmbH}$ die Automatisierungstechnik des Bereiches Engineering und ist Leiter des Evonik-Arbeitskreises für Funktionale Sicherheit. Die Verknüpfung zwischen Cyber-Security und Funktionaler Sicherheit ist Bestandteil des Aufgabengebietes.

Dr. MICHAEL KRAUSS (geb. 1984) ist seit seiner Promotion in theoretischer Physik 2010 im Fachzentrum für Automatisierung der BASF SE in
Ludwigshafen tätig. Ab dem Jahr 2013 leitete er ein Fachgebiet für Prozessleitsysteme mit dem Fokus auf Automatisierungsprojekten. Seit 2018 leitet er in der Funktion als Senior Automation Manager und Senior Expert das Gebiet Control Systems Technology. 2014 übernahm er in der NAMUR die Leitung der Arbeitskreise 2.1 „PLS“ sowie 4.20 „Remote Operation“.

Dipl.-Ing. UDO MENCK (geb. 1966) ist in einer globalen Abteilung für Safety Instrumented Systems bei Dow Chemical in Stade tätig. In seiner Funktion als „Global Functional Safety Manager“ betreut er weltweit die Implementierung von Sicherheitsfunktionen mit Hilfe von Prozessleittechnik unter Berücksichtigung aktueller Regelwerke und den Herausforderungen aus dem Bereich Cyber Security.

Dr. GREGOR SCHMITT-PAUKSZTAT (geb. 1977) leitet die Gruppe Modeling \& Technical Safety im Bereich Process \& Plant Safety bei Bayer. In dieser Funktion koordiniert er unter anderem das Thema ,Funktionale Sicherheit‘ in der Region EMEA.

Bayer AG,

Kaiser-Wilhelm-Allee,

51368 Leverkusen,

Tel. +49 (0) 2143043692 ,

E-Mail: gregor.schmitt-pauksztat@bayer.com 